\title{
Gas-Diffusion-Layer Structural Properties under Compression via X-Ray Tomography
}

\author{
Iryna V. Zenyuk ${ }^{\mathrm{a}^{*}}$, Dilworth Y. Parkinson ${ }^{\mathrm{b}}$, Liam G. Connolly and Adam Z. Weber ${ }^{\mathrm{c}}$

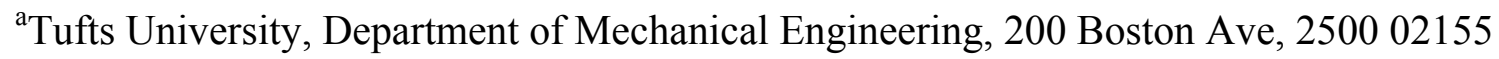 \\ ${ }^{\mathrm{b}}$ Advanced Light Sourceand ${ }^{\mathrm{c}}$ Energy Conversion Group, Energy Technologies Area, Lawrence \\ Berkeley National Laboratory, 1 Cyclotron Road, Berkeley CA 94720, USA \\ *Corresponding author: e-mail: Iryna.Zeyuk@tufts.edu, tel.: +1(617)627-7956
}

\begin{abstract}
There is a need to understand the structure properties of gas-diffusion layers (GDLs) in order to optimize their performance in various electrochemical devices. This information is important for mathematical modelers, experimentalists, and designers. In this article, a comprehensive study of a large set of commercially available GDLs' porosity, tortuosity, and pore-size distribution under varying compression is presented in a single study using X-ray computed tomography (CT), which allows for a noninvasive measurement. Porosities and PSDsare directly obtained from reconstructed stacks of images, whereas tortuosity is computed with a finite-element simulation. Bimodal PSDsdue to the presence of binder are observed for most of the GDLs, approaching unimodal distributions at high compressions. Sample to sample variability is conducted to show that morphological properties hold across various locations. Tortuosity values are the lowest for MRC and Freudenberg, highest for TGP, and somewhere in-between for SGL papers. The exponents for the MRC and Freudenberg tortuosity have very small dependence on compression because the shapes of the pores are spherical indicating minimal heterogeneity. From the representative elementary volume studies it is shown that volumes of $1 \times 1 \mathrm{~mm}$ in-plane and full thickness in through-plane directions accurately represent GDL properties.
\end{abstract}


Keywords: fuel-cells, gas diffusion layers, porosity, pore-size distribution, tortuosity, compression 


\section{Introduction}

Gas-diffusion layers (GDLs) find application in various electrochemical technologies including polymer-electrolyte fuel cells (PEFCs) and redox flow batteries (RFBs) [1-8]. For these devices to reach commercialization, cost reduction and performance optimization is required $[1,3]$. PEFCs with novel thin-film electrodes, such as 3M's nano-structured thin-film (NSTF) electrodes have reduced platinum (Pt) electrocatalyst loading and have demonstrated high power-densities; however, water-management during start-up and at lower temperatures is a significant challenge [1, 9-13]. In PEFCs, water is formed in the cathode and is removed through porous GDLs that provide structural support for membrane electrode assembly, delivery of gas reactants, electron and heat transport, and removal of product water $[14,15]$. In RFBs, they also have the added functionality of providing a catalytic surface [5-7]. Understanding morphology and transport properties of these porous layers is essential for optimizing water management, mass transport, and performance.

GDLs are fabricated using polyacrylonitrile (PAN) polymer, which is carbonized by heating in a range of 1200 to $1350^{\circ} \mathrm{C}$ in nitrogen [16]. For most of the GDLs, the polymer matrix is bound together chemically with carbonaceous binder (paper GDLs) or mechanically (woven GDLs). The resulting carbon fibers have diameters of 6 to $8 \mu \mathrm{m}$ and wide pore-size distribution (PSD) with a mean radius in the range of tens of micrometers [16]. Commercial GDLs have a thickness in the range of 190 to $440 \mu \mathrm{m}$ and are anisotropic because of fiber preferential in-plane orientation during roll-to-roll processing. In the last fabrication step, the GDL is made hydrophobic, typically by infiltration of polytetrafluoroethylene (PTFE) or perhaps by direct fluorination [4]. 
Various morphological metrics can be used to characterize the structure of GDLs and effective transport parameters. Porosity, PSD, and tortuosity are three major characteristics, from which effective transport properties can be derived indirectly [17-20]. Porosity is defined as a ratio of void volume to total volume and it is relevant for gaseous and water transport. Knowledge of porosity is needed to model gas transport and liquid permeability accurately as effective diffusivity and permeability are directly proportional to it $[18,21]$. Many experimental techniques have been developed to measure GDL porosity, which is challenging due to their thin, compressible, and inhomogeneous geometry $[22,23]$. These techniques include gas pycnometry [22, 23], mercury intrusion porosimetry (MIP)[24-26], weighing [17, 27], and buoyancy [20]. A detailed comparison of these methods is given by Rashapov et al. [20], where shortcomings of the methods are mainly due to the inability to resolve the interfacial porosity properly, and limitations of these techniques for a single, thin GDL layer. Moreover, these techniques are limited for measuring the porosity of a free-standing GDL and cannot be applied for compressed GDLs, as they exist in assembled cells. For example, to estimate the porosity of a compressed GDL, empirical relationships are used that linearly interpolate or extrapolate compressed porosities assuming the solid volume fraction does not change [17, 23].

PSDs are useful for obtaining water-retention curves [24, 28], which are plots of capillary pressure vs. liquid-water saturation. For hydrophobic media, a non-wetting fluid will intrude voids with larger pore diameter and progressively fill the smaller voids at higher capillary pressures. Therefore, knowing GDL PSDs is essential in predicting water transport through these layers. MIP is the most common method of measuring PSDs of GDLs, where a sample is submerged in mercury (completely non wetting) and at each increasing capillary pressure mercury is forced into smaller pores [24-26]. Thus, capillary pressure and volume are recorded 
and the PSD obtained. One of the challenges of this method is the necking effect, where a large void behind a narrow neck is mistakenly attributed to a volume of a narrow pore. There is a need for an alternative method for PSD that is more reliable and non-invasive.

A GDL's tortuosity is generally extracted from measurements of effective gas diffusivity. A variety of experimental set-ups have been developed to study the diffusivity of the GDLs [17-19, 29-34]. These include a Loschmidt cell, electrochemical impedance spectroscopy, and gradient methods (e.g., water vapor, limiting current density, or local chemical sensors). These techniques have been mostly applied to study conventional GDLs and primarily concentrated on throughplane effective diffusivity. Effective media theories and computational models exist as well to predict gas transport through GDLs [28, 35-41]. Several recent efforts extended Bruggeman's theory, which is applicable for homogeneous porous media with spherical voids or solids, to represent a GDL's inhomogeneity by allowing for a number of particle shapes and orientation anisotropy [40, 42]. Many of the computational efforts concentrate on generating a GDLs structure stochastically by using bulk properties such as porosity, fiber diameter, and percentage of binder as fitting parameters [36, 41, 43]. Numerical models are then applied to these generated structures to extract effective transport properties. Alternatively, a selected class of models utilized direct reconstructions of the GDL domains from available three-dimensional X-ray CT images [37, 38, 44].

X-ray CT allows one to resolve the internal structure and inhomogeneity under controlled conditions including compression $[14,39,45-53]$. This technique is straight-forward and virtually error-free as the only error is due to a threshold selection that separates pore from fiber and binder. To the best of authors' knowledge, X-ray CT is the only feasible technique to resolve porosity and pore structures under compression and at various regions within the GDL (either in 
plane or through thickness). This technique has been used to measure GDL properties in limited studies including spatially-resolvedporosity [50, 52, 54, 55], effects of compression [14, 53, 54] and water distribution [37][14, 49, 52]. Many of these studies focused on a single GDL or property, and a comprehensive comparative study with various information is missing. Moreover, differential PSDs for GDLs under various compressions have not been reported previously.

In this paper, we use X-ray CT and computational models to characterize the morphology of various commercial GDLs under different levels of compression and PTFE loading to uncover underlying relationships and trends and provide data for modeling and GDL designers including spatial porosity, PSD, and ellipsoid factor. In addition, we also explore the issue of representative elementary volumes (REVs) for GDLs, which is the smallest volume of the sample that retains the bulk properties [56], a critical parameter in terms of analysis and utilization that cuts across various experiments and theory.

\section{Methods and Materials}

\subsection{Experimental Setup}

The tomography experiments for the GDL characterization were conducted at Beamline 8.3.2 at the Advanced Light Source (ALS). A double-multilayer monochromator was used to select 14 $\mathrm{keV}$ X-rays, and detection was with a $0.5 \mathrm{~mm}$ LuAG scintillator and 5x lenses with a sCMOS PCO.Edge camera, giving a $1.33 \mu \mathrm{m}$ pixel dimension, and a $3.3 \mathrm{~mm}$ horizontal field of view (FOV). Using a $300 \mathrm{~ms}$ exposure time yielded 8000 countson the 16-bit camera (allowing a 
maximum of 65535 counts).For each tomographic scan, 1025 projections were acquired over a $180^{\circ}$ rotation, within scan-time of $7 \mathrm{~min}$.

The schematic of the sample holder is shown by Figure 1. The sample-holder used for tomography consists of an aluminum stage, and a high X-ray transmitting Vespel® cup with wall thickness less than $1 \mathrm{~mm}$. A $3.2 \mathrm{~mm}$ diameter circular GDL sample was cut and pressed against the aluminum stage with a flat stamp. After the sample was mounted on the stage, an ultra-fine pitch thread was used to control GDL compression.

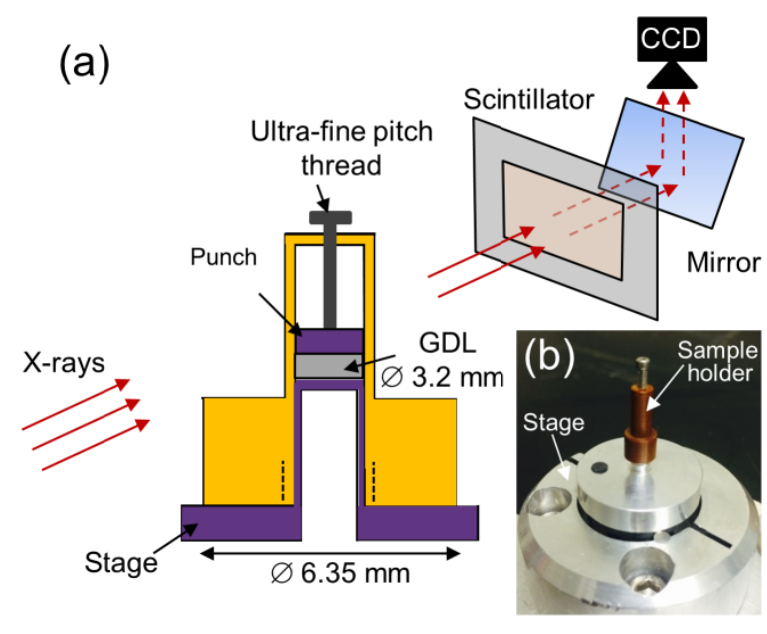

Figure $1 \mathrm{a})$ A schematic of sample-holder set-up at the beamline and b) a photograph of the sample holder mounted on the stage.

\subsection{Materials}

The carbon papers investigated in this work are TGP 060 and TGP 120 (Toray International) with three levels of PTFE loading - 5, 10 and 20\%, SGL (SIGRACET®, SGL Carbon Inc.) series 10 BA, 24 AA, 24 BA, 25 AA, 35 AA, 35 BA, MRC 105 (Mitsubishi Rayon Corp.), and H2315 (Freudenberg), and several with microporous layers (MPLs): TGP 060 5\% PTFE, SGL 10BC, SGL 24 BC, SGL 25 BC, SGL 35 BC. 


\subsection{Image Reconstruction and Segmentation}

Preprocessing of the acquired images was performed with Fiji/ImageJ, then phase retrieval was performed with the Modified Bronnikov Algorithm (MBA), and tomographic reconstruction (including a ring reduction filter) was performed using Octopus 8.6 [57]. Image segmentation and analysis was carried out with Fiji/ImageJ. Two phases were identified during segmentation: fiber and void space, and these were separated by thresholding, with the threshold determined by the Otsu algorithm. Details of the grey-level histograms, and samples of segmented tomograms are shown in Supplementary Info (SI). For the samples with a microporous layer, a 3D median smoothing filter with a radius of 5 pixels was chosen. Compressed GDL thickness was measured in seven selected locations and average and standard deviation was reported. The image was cropped manually to avoid larger interfacial pores.

\subsection{Morphological Properties}

\subsubsection{Porosity and PSDs}

Porosity was computed with Fiji/ImageJ using the binary histogram for the segmented image. The spatial-distribution of porosity for different directions was computed with Avizo Fire 8.1 based on the segmented image. For porosity calculation $2.2 \times 2.2 \mathrm{~mm}$ samples and various thicknesses (depending on compression) were broken down into four $1.1 \times 1.1 \mathrm{~mm}$ quarters, where average porosity and standard deviation were reported. Different methods exist in the literature for computing PSDs from segmented image-stacks using a variety of commercial and open-source software. Here we extracted the PSDs with in the "Local Thickness" Fiji/ImageJ plugin[58]. Local thickness is defined as the diameter of the largest sphere that fits inside the object and contains the point, $\vec{p}$, as discussed in the SI: 


$$
t(\vec{p})=2 \max (\{r \mid \vec{p} \in \operatorname{sph}(\vec{x}, y) \subseteq \Omega, \vec{x} \in \Omega\})[1]
$$

\subsubsection{Tortuosity}

Tortuosity here is defined as a ratio of the actual path length, $L_{e}$, to the Euclidean distance, $L$, squared: $\tau=\left(L_{e} / L\right)^{2}$ [59]. To quantify the transport-dependent GDL morphological properties, a tortuosity is calculated,

$$
\tau=\frac{\varepsilon}{D_{e f f} / D}=\frac{\varepsilon}{K_{f}}[2]
$$

where $\varepsilon$ is the porosity, $K_{f}$ is the formation factor, $D$ is the intrinsic diffusion coefficient, and $D_{\text {eff }}$ is the effective diffusion coefficient determined by

$$
D_{e f f} / D=\frac{N}{A} \frac{L}{\Delta c}[3]
$$

where $N$ is the superficial flux, $A$ is the in-plane GDL area, $L$ is the GDL thickness, and $\Delta c$ is the concentration difference between the inlet and outlet (assumed to be $\Delta c=1$ for the simulations). To calculate the tortuosity or formation factor, we set the diffusion coefficient arbitrarily to 1 and solve a finite-element simulation through the GDL using boundary conditions of $c=1$ and $c=0$ for the GDL through-thickness boundaries, respectively. A no-flux condition is used for the other boundaries.

To enact the simulation, computational realization of the GDL is required. LatticeBoltzmann (LB) method or computational fluid dynamics (CFD) can be used to determine tortuosity and formation factor of the GDLs. Both methods are widely used in the community, and here only salient comparison details are given; for a more in-depth review the reader is 
referred to reference [60]. LB method is relatively simple to implement, as the physical domains are represented with lattice of 3D image voxel grid and there is no need for computationallyexpensive meshing, it is suitable for parallelization [61], and especially useful for tracking phase boundaries for multiphase flow. Traditional CFD methods are numerically efficient for singlephase flow, however, some difficulties are encountered when simulating multiphase flow. While both methods have been shown to be applicable and provide comparable results in the solution of steady-state transport in porous media $[62,63]$, herein we chose to use CFD for calculation of tortuosity for the single-phase, steady-state tortuosity.

There are several ways to represent computationally the GDL domain generated through the image-binarization process. The domain can be meshed directly; however, this method is computationally intensive, especially for a GDL, which features long fibers that requires a significant level of detail. An alternative approach is to generate stochastically the fibers in the domain, which is less computationally expensive; however, this method is not deterministic. Here, we select a third approach, where we assign local diffusivities to 0 if it is fiber and to 1 if it is void. Due to large pore sizes, Knudsen diffusion was neglected.

The finite-element simulations were performed using Comsol Multiphysics 5.1 (COMSOL, Inc., Burlington, MA). LiveLink to Matlab was used to map physical domain space to diffusion coefficient matrix. A 3-D binary image was stored onto a Matlab workspace that represented GDL with various thicknesses depending on compression. A domain was created in Comsol with the same dimensions as the binary image in Matlab and a mesh was generated on this domain. Comsol passed a vector of $\mathrm{x}, \mathrm{y}$ and $\mathrm{z}$ coordinates onto Matlab and Matlab returned a vector of diffusion values (0s or 1s). Interp3 function was used to interpolate Comsol's spatial vector onto diffusivity matrix. A mesh-conversion study was performed to select an optimal 
mesh-size $\left(0.18 \mu \mathrm{m}^{-3}\right.$ mesh density), where tradeoffs of computational error vs. time have to be considered as described in the SI. Overall, the study spanned a total 210 simulations. Detailed information for simulations and mesh-convergence study are provided in SI.

\section{Results and Discussion}

In the following sections, the various structural properties are characterized, although first a discussion on representative volumes is made to ensure data reproducibility and examine GDL heterogeneity.

\subsection{Porosity}

Figure 2 shows porosity of a) SGL, b) Toray and c) MRC and Freudenberg GDL samples for various compressions, where strain is computed with respect to in-house measured GDL thickness as reported in Table 2.Figure 2a shows porosity of the SGL series as a function of strain. Porosity for the least compressed GDLs varies from 0.7 to 0.86 . SGL 24 AA and 24 BA showed the largest drop in porosity with compression. For example, SGL 24 BA porosity changes from 0.77 to 0.57 for strains of 0.02 and 0.4 , respectively. Figure $2 \mathrm{~b}$ shows the porosity distribution for TGP 060 and 120 series with 5, 10 and 20\% PTFE. For compression less than 10 $\%$, most of these GDLs had porosities between 0.6 and 0.7 . Both TGP 060 and 120 with $20 \%$ PTFE showed the lowest porosity out of all of the GDLs. Figure $2 \mathrm{c}$ shows the porosity as a function of strain for MRC 105 and Freudenberg H2315, where for the least compressed samples, the porosity was 0.72 and 0.68 , respectively. Comparing the various GDLs, the average porosity in decreasing order is: $\mathrm{SGL}>\mathrm{TGP}>\mathrm{MRC} \approx$ Freudenberg. 
The porosity obtained with X-ray CT technique is compared to that available in literature in Table 1. It is worth noting that for TGP120, with three degrees of PTFE content $(5,10$, and $20 \%$ ), literature data does not show a clear indication that porosity has decreased with increase in PTFE content. The reason for this is that PTFE distribution is inhomogeneous. Rashapov et al. [20] used simple expression based on sample total mass balance and showed that increasing PTFE content from 0 to $20 \%$ resulted in a decrease of porosity from 0.75 to 0.7 for TGP 060 and 0.78 to 0.72 for TGP 120 . This decrement is close to that observed inthe X-ray CT data. The porosities reported for TGP 120 herein are also consistent with those obtained by KhajehHosseini-Dalasm et al. [53], however, they are lower compared to the porosity obtained with MIP $[25,64]$ and boyancy methods [20]. The literature reportedporosity for TGP060 with 10 and 20\% PTFE ranges from 0.67 to 0.75 , which is higher than that measured here, although here it was under compression. The porosities for the other samples compare favorably with literature data where available.

The data from Figure 2 were fit with a linear regression:

$$
\varepsilon=\varepsilon_{0}-a \eta\left(1-\varepsilon_{0}\right)[4]
$$

where $\varepsilon_{0}$ is extrapolated porosity for zero compression, $\eta$ is the strain, and $a$ is a fitting parameter. The various fit parameters are tabulated in Table 2. This fit equation is similar to that of Fluckiger et al.,[17] but the use of X-ray CT allows for a more accurate measurement of porosity under compression, As can be seen from Table 2, the effective slope, a, varies and essentially relates the dependence of porosity with compression. From the study, it is concluded that the SGL papers have the largest reduction in porosity with compression. 

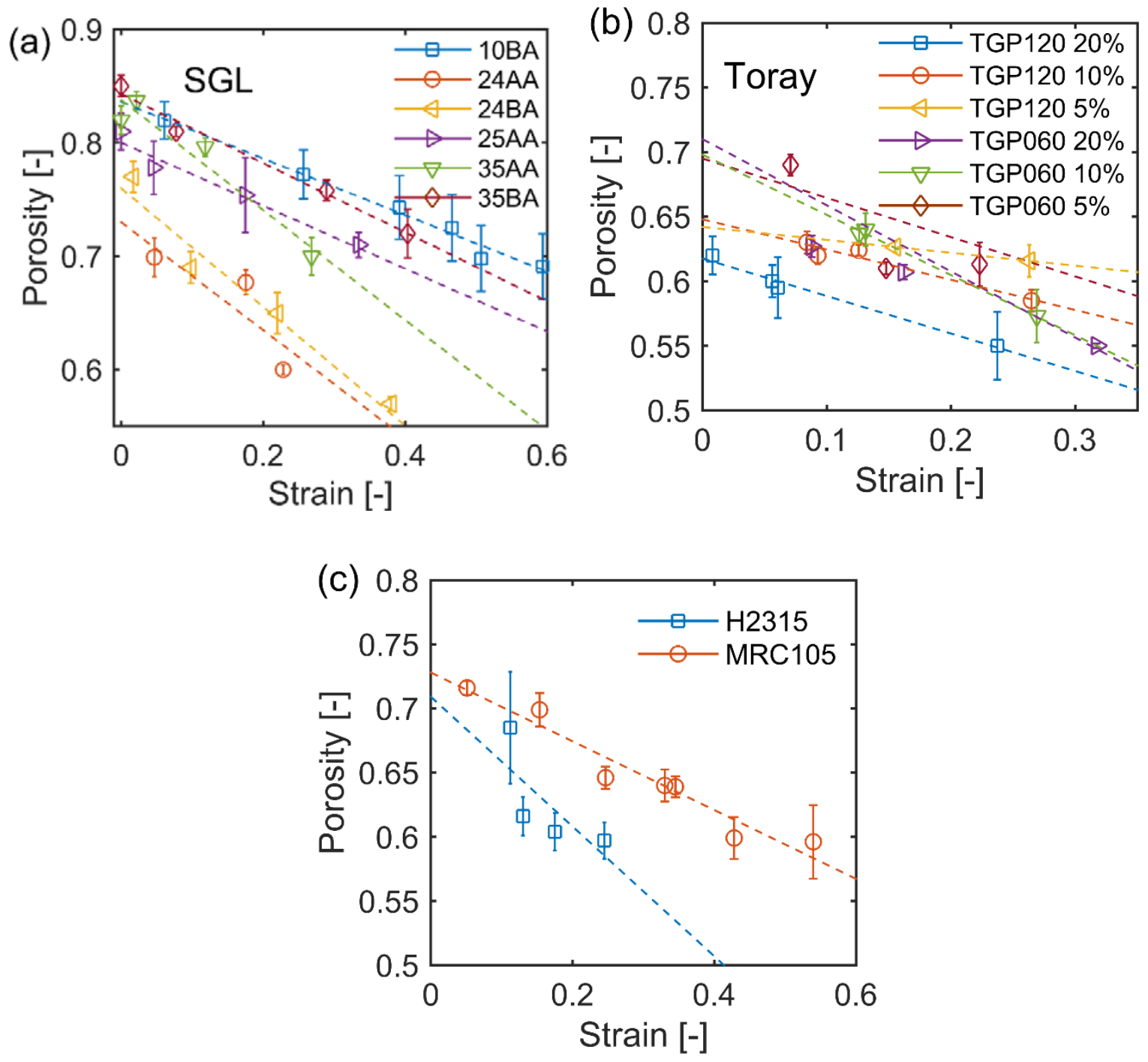

Figure 2. Porosity as a function of compression (strain) for a) SGL, b) Toray and c) H2315 and MRC 105 GDLs. The dashed lines are fits to the experimental data using Eq. 6 as reported in Table 2 .

\subsection{Pore-size distribution}

\subsection{1. $X$-ray CT PSDs}

PSD can be represented in terms of count, normalized volume of GDL or as a power density function (PDF). In this paper, we refer to PSD obtained with PDF as described below and these two terms are used interchangeably. Figure 3 shows PSDs generated for different GDLs for 
various levels of compression; the differential between PSD of the distribution with compression. The fits to the PDFs were generated using a log-normal bi-modal PSD:

$$
\operatorname{PDF}(r)=\sum_{k=1,2} f_{r, k}\left\{\frac{1}{r \sigma_{k} \sqrt{2 \pi}} \exp \left[-\frac{\left(\ln r-\ln r_{0, k}\right)^{2}}{2 \sigma_{k}^{2}}\right]\right\} \quad[5]
$$

where $\operatorname{PDF}(r)$ is the normalized volume of pores having radius $r, f_{r, k}$ is the fraction of pores that make up the distribution $k$, and $r_{0, k}$ and $\sigma_{k}$ are the characteristic radius and spread of distribution $k$, respectively. The fits were generated for all of the GDLs and the fit parameters for SGL 25AA, TGP 120, 20\% PTFE, H2315 and MRC 105 are reported in Table 3; the PSDs for the remaining GDLs and fits can be found in the SI.

Figure 3a shows the PDF for a SGL 25AA at compressions of $0,4.6,17.5$ and $33.4 \%$. Clear bimodal distributions are observed for all four levels of compression, where the smaller and larger radii for this distribution are reported in Table 3. The peak that corresponds to larger radii shifts to smaller values, from 39.8 to $25.2 \mu \mathrm{m}$ at compression 0 to $33.4 \%$, respectively, whereas the peak corresponding to the smaller mean radius does not change significantly (shifts from 13.7 to $12.2 \mu \mathrm{m})$. Thus, the distance between the twomean radii decreases with compression, showing that it is the larger pores that are compressed. This is seen better in the right plot, which shows the $\triangle \mathrm{PDF}$ between the compressed and uncompressed PDFs. Here, the larger pores become smaller with compression. A transition point is observed for all three compressions; for SGL 25AA it is 30 to $35 \mu \mathrm{m}$. Similar bimodal distributions with large mean radii are observed for all SGL samples as reported in the SI. 
As shown in Figure 3b, the PDF for TGP 120 with 20\% PTFE is also bimodal but the mean radii are closer together compared to the SGL 25AA GDL. From Table 3, for uncompressed samples, the mean radii are 9.57 and $14.7 \mu \mathrm{m}$, whereas for the compressed GDL at $23.7 \%$, the radii become 9.27 and $13.7 \mu \mathrm{m}$, respectively. This difference is about 5 times smaller compared to the SGL 25AA sample. From the $\triangle \mathrm{PDF}$ plot, the transition radius is about $18 \mu \mathrm{m}$. The PSDs for TGP 120 and TGP 60 for PTFE wetting of 5, 10 and 20\% are reported in SI and all show similar PSD to the one shown by Figure $3 \mathrm{~b}$.

The PSD for Freudenberg H2315 for four levels of compression is shown in Figure 3c. Unlike the previous samples, a unimodal distribution is realized. As seen in Table 3, the mean radius of the distribution decreased from 9.5 to $7.28 \mu \mathrm{m}$ as compression increased from 11 to 24\%. This mean radius is smaller than the mean radii for SGL 25AA and TGP120 for similar levels of compression. From the plot on the right, the transition radius for $\Delta \mathrm{PDF}$ is $10 \mu \mathrm{m}$, indicating that the percentage of volume for the pores carrying this radius does not change as compression increases.

The PSD for MRC 105 is shown by Figure 3d for seven levels of compression, varying from 5 to $53 \%$. For five of these compressions, a bimodal PSD is observed, whereas for the remaining two a unimodal distribution occurs. The mean radii of distribution for MRC 105 were closer to those of Freudenberg H2315 GDL, the larger radius decreased from 13.09 to $7.37 \mu \mathrm{m}$, as compression increased from 5 to $53 \%$. The transition radius is similar to that observed in H2315 GDL and fluctuates around $10 \mu \mathrm{m}$. A comparison for PSDs with MIP was done, where good agreement was observed between MIP and tomography for mean radii, as shown in SI, Figure S5. Moreover, a sample-to-sample variability study was conducted, where the PSDs for 
five MRC 105 and SGL 10 BA cut at various locations showed good agreement, as shown in SI, Figure S26. 

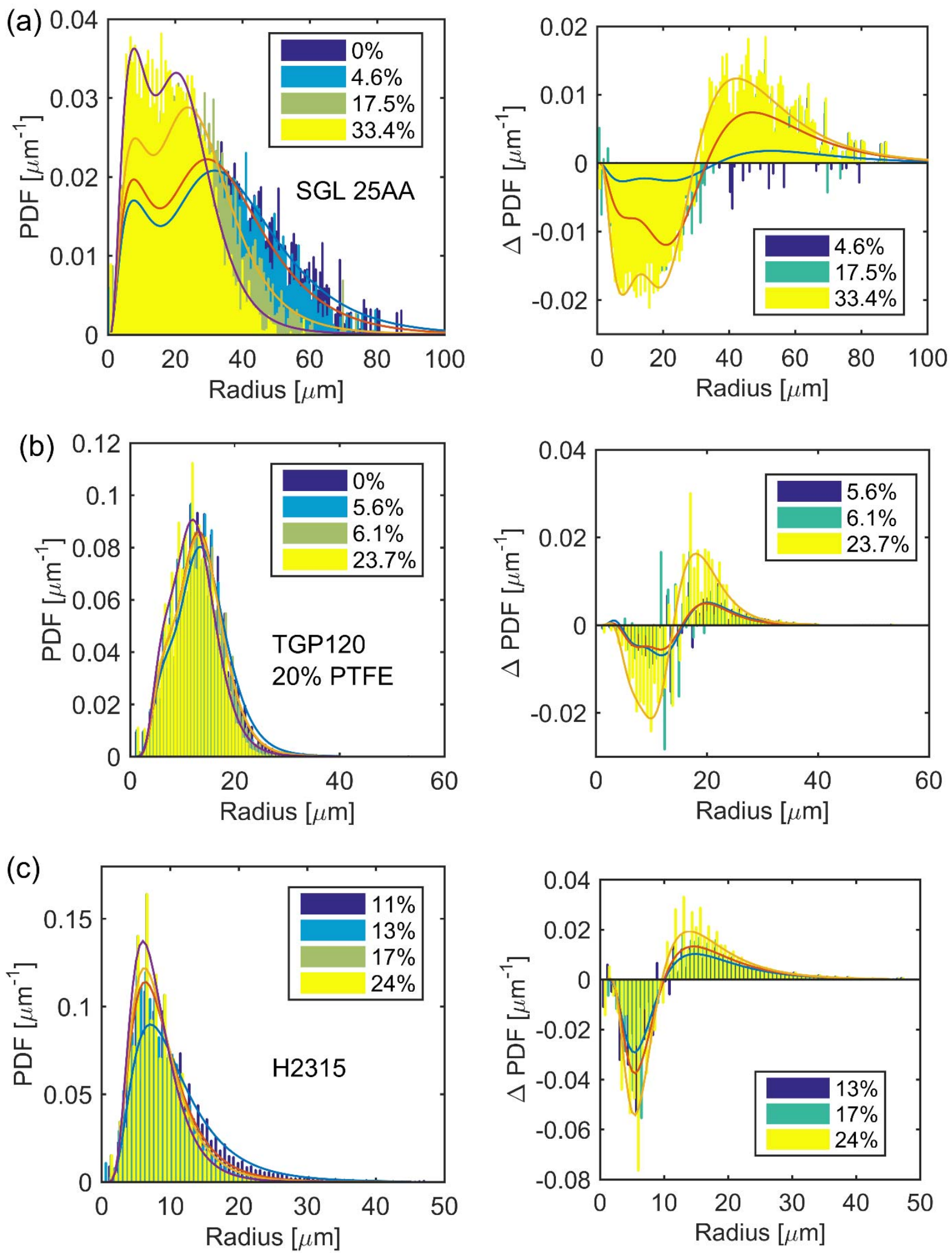

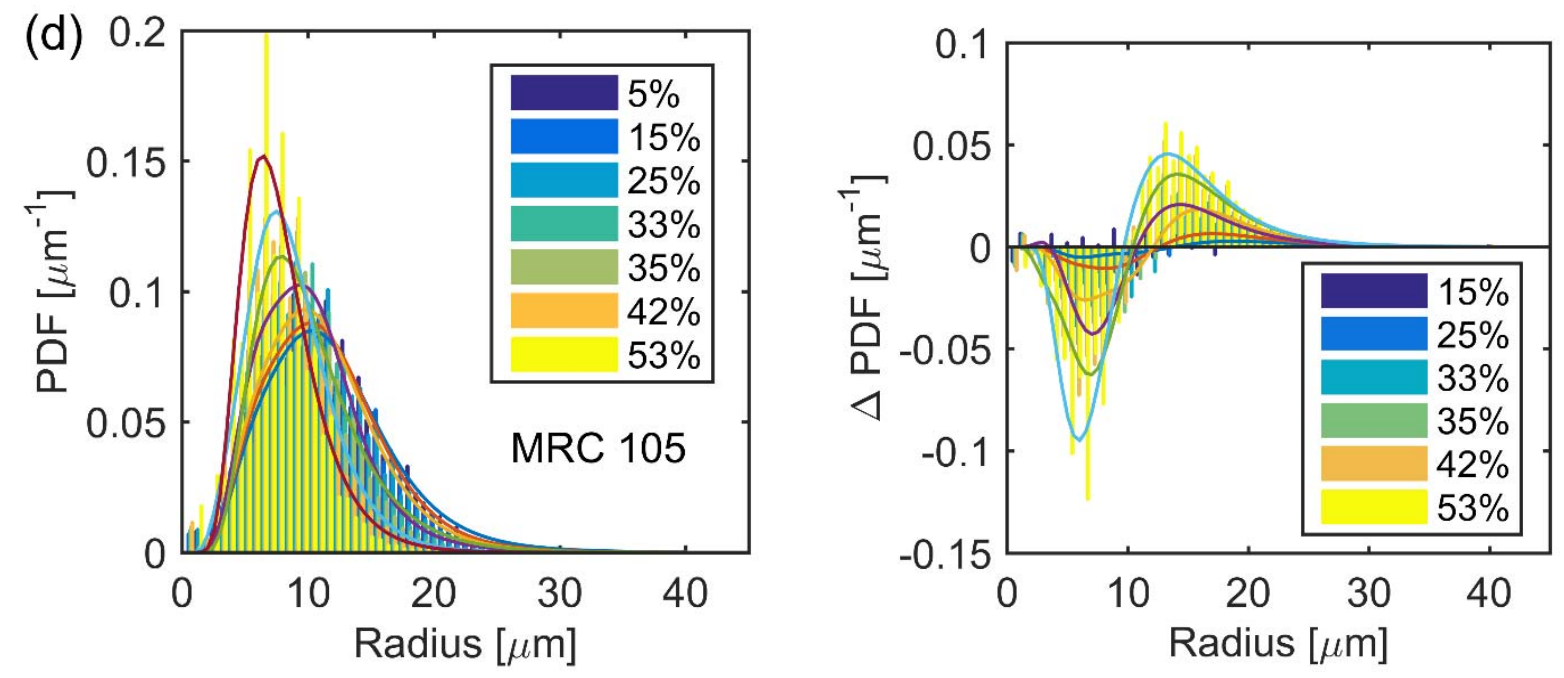

Figure 3. PSDs for different levels of compression for a) SGL 25AA, b) TGP120 20\% PTFE,c) $\mathrm{H} 2315$, d) MRC 105, where $\triangle \mathrm{PDF}$ plots represent the difference in the volume between the least compressed and that at higher compressions.

To explore the different GDLs, a single mathematical mean radius was obtained for each PSD and plotted inFigure 4 as a function of strain. This average pore radius, $r_{\text {mean }}$, was determined from the PDF: $r_{\text {mean }}=\int_{0}^{r_{\max }} r \times P D F(r) d r$, where the integration was taken over all existing radii. The maximum radius of inscribed spherical elements for each GDL was also reported and plotted in SI. For the SGL samples, a linear decrease in mean radius with compression is observed as shown by Figure 4a. Series 10 and 35 showed higher mean radius for a given compression compared to series 24 and 25, where the 10 and 35 SGL series are thicker and contain larger pores primarily in the middle of the GDL [65]. The mean radii for GDLs with MPLs are about $10 \mu \mathrm{m}$ smaller compared to the same GDLs without MPLs. Because micro Xray CT does not resolve nano-sized pores in the MPLs, the MPL was considered to be solid in this analysis; therefore, these results over-approximate MPL's reduction of mean radius. 
For the TGP GDLs, there is a strong similarity of mean radii (see Figure 4b), which is about half as large as the SGL ones. For the same PTFE content, TGP 120 and TGP 060 should have the same radii as the only nominal difference between these GDLs is their thickness. The mean radii for various MRC105 and Freudenberg H2315 are shown in Figure 4c. For uncompressed MRC, the mean radius is $12 \mu \mathrm{m}$ and it decreases to $7.5 \mu \mathrm{m}$ for a compression of 53\%. For Freudenberg H2315, the mean radius decreased from 10.2 to $7.3 \mu \mathrm{m}$ as compression increased from $11.2 \%$ to $24.5 \%$, respectively

For ease of analysis, use, and comparison, the linear regression fits for the mean radii as a function of compression were determined,

$$
r_{\text {mean }}=r_{0, \text { mean }}-a_{\text {mean }} \eta[6]
$$

where $r_{\text {mean }}, r_{0, \text { mean }}$ are computed mean radius and interpolated mean radius at 0 strain, respectively, and $a_{\text {mean }}$ is a slope. The values of $r_{0, \text { mean }}$ and $a_{\text {mean }}$ are reported in Table 2. Similar linear expression was used to fit maximum radii as functions of strain:

$$
r_{\max }=r_{0, \max }-a_{\max } \eta[7]
$$

where values of $r_{0, \max }$ and $a_{\max }$ are reported in Table 2. These linear regression fits can be directly used in the studies where porosity is not known but only compression or strain is measured. 

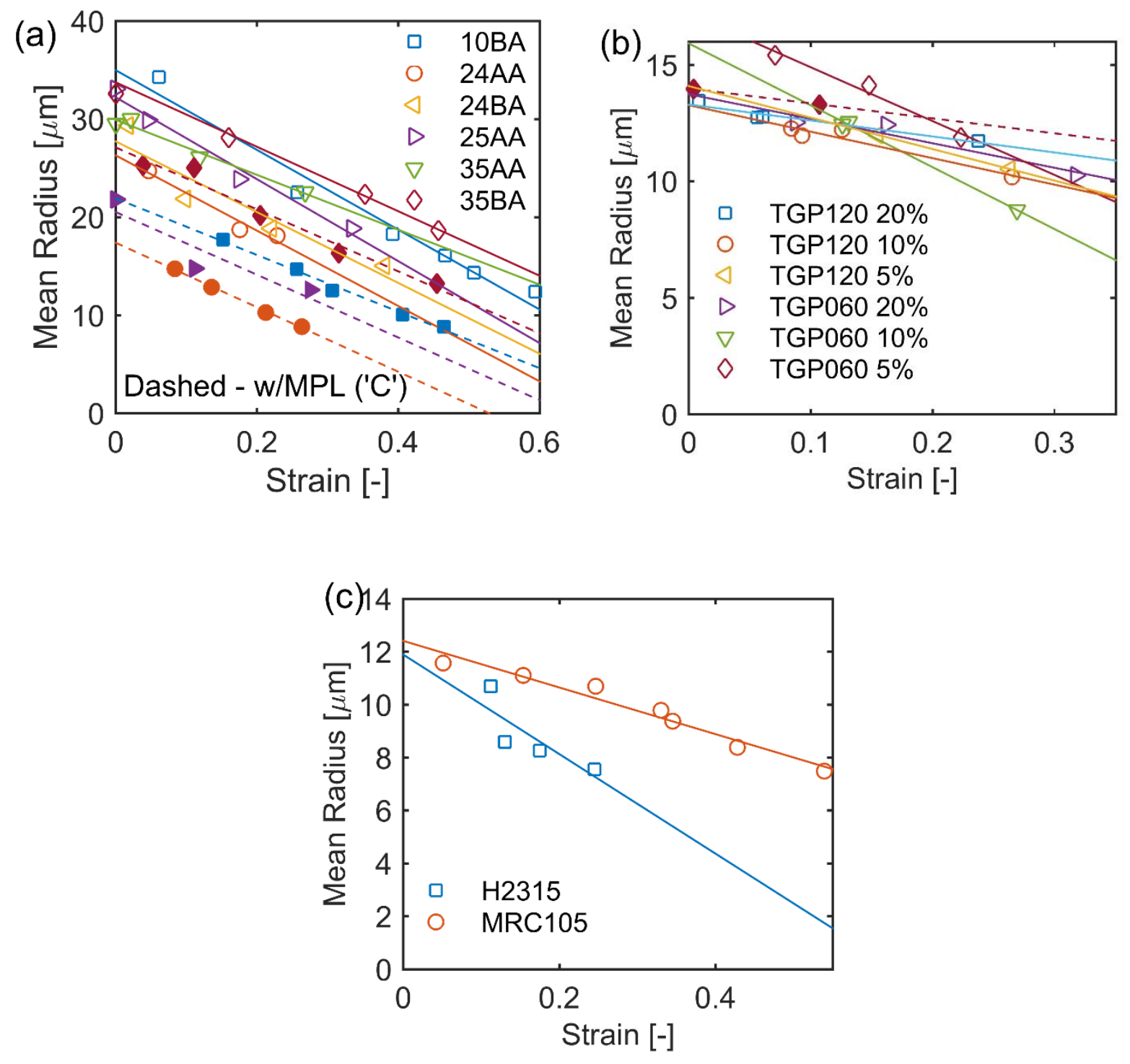

Figure 4 Mean radii from PSDs for a) SGL, b) TGP and c) H2315 and MRC 105 GDLs as functions of strain. Solid symbols show GDLs with MPLs. Linear regression fits are also shown, where the slope and intercept are reported by Table 2 .

\subsection{Tortuosity}

The tortuosity is most often cast as a function of porosity, where the porosity is changing herein due to compression. Figure 5 shows the calculated formation factors as functions of porosity (the tortuosity plots are shown in SI). Figure 5a shows formation factors as functions of 
porosity for SGL series GDLs. For the SGL GDLs, the tortuosity values varied from 1.5 for the least compressed samples (highest porosity) to 3 for the most compressed one. SGL 10 BA showed the lowest tortuosity values, and overall there was little deviation amongst the different GDLs. The formation factor curve was fit with the power-law relation for porosity,

$$
f(\varepsilon)=\varepsilon^{m}[8]
$$

where the average exponent, $m$, was 2.8. This fit is somewhere in-between a value of $m=3.8$ and the pore-network model expressed by Tomadakis and Sotirchos [21] for fibrous structures,

$$
f(\varepsilon)=\left(\frac{\varepsilon-\varepsilon_{p}}{\varepsilon_{0}-\varepsilon_{p}}\right)^{\alpha}[9]
$$

where $\varepsilon_{p}$ is the percolation threshold, and for through-plane diffusion the values for $\varepsilon_{p}$ and $\alpha$ were 0.11 and 0.785 , respectively. The results for SGLs presented here for tortuosity and formation factor are in excellent agreement with the experimental studies by Hwang et al. [18] and Most et al. [23], however deviate significantly from Fluckiger et al. [17]. This latter study used the analogy between Fick's and Ohm's laws to calculate the formation factor from electrochemical impedance-spectroscopy data of ionic solutions in the porous media. For uncompressed GDLs, MIP or a similar technique was used to determine porosity. For compressed GDLs (lower porosities), the porosity was computed with an analytical fit by the two studies, which as discussed in Section 3.2, results in errors due to the nonlinear change in porosity with strain.

Formation factors for TGP 060 and 120 for PTFE loadings 0-20\% are shown in Figure $5 \mathrm{~b}$, where the tortuosity values (shown in SI) range from 1.5 to 3.5 as porosity decreases from 
0.72 to 0.5 . As expected, higher tortuosities were observed for GDLs with 20\% PTFE content compared to those of 5 and $10 \%$. Comparing to literature data where available, the tortuosity values reported here are lower by 1 at a porosity of 0.7 and less than that for lower porosity values. Good agreement with Baker et al. [34] is observed for TGP 060 and porosity range of 0.65 to 0.5 . The disagreement with the other experimental data is primarily due to a method of calculating porosity that the other studies use and also perhaps due to effects of lands and channels geometry in-situstudies. Our reported porosities for the lowest degree of compression are generally lower by 0.1 compared to those in literature using different analysis methods.

Finally, Figure 5c shows formation factors for MRC 105 and H2315. Both GDLs show similar tortuosity values for the range of porosity that varied between 1.4 to 1.75 (see SI). These values are in good agreement with Totzke et al. [54], who used X-ray CT technique for H2315 tortuosity estimation. For uncompressed H2315, Hwang et al. [18] reported a tortuosity value that was 0.8 higher than that obtained from tomography studies, which is possibly due to extra distance that gas has to travel in the in-situassembly. The formation factor curve is best represented with porosity to an exponent of 1.9 , which is lower than that for SGL and Toray GDLs.

Table 4 shows formation factor exponents, $m$, for individual GDLs; these fits are collapsed into one for Figure 5. In the SGL series, 10 BA shows the lowest exponent, $m=2.28$ and hence has the lowest tortuosity, whereas 24AA shows the highest exponent, $m=2.8$. In the TGP series, $m$ increased with increase in PTFE weight, with the largest $(m=3)$ for TGP120, PTFE 20\%. As mentioned earlier, MRC and H2315 have the lowest tortuosities, with $m=1.9$ and 1.83 for MRC 105 and $\mathrm{H} 2315$, respectively. The table also shows reported exponents from literature that are also plotted in Figure 5. 

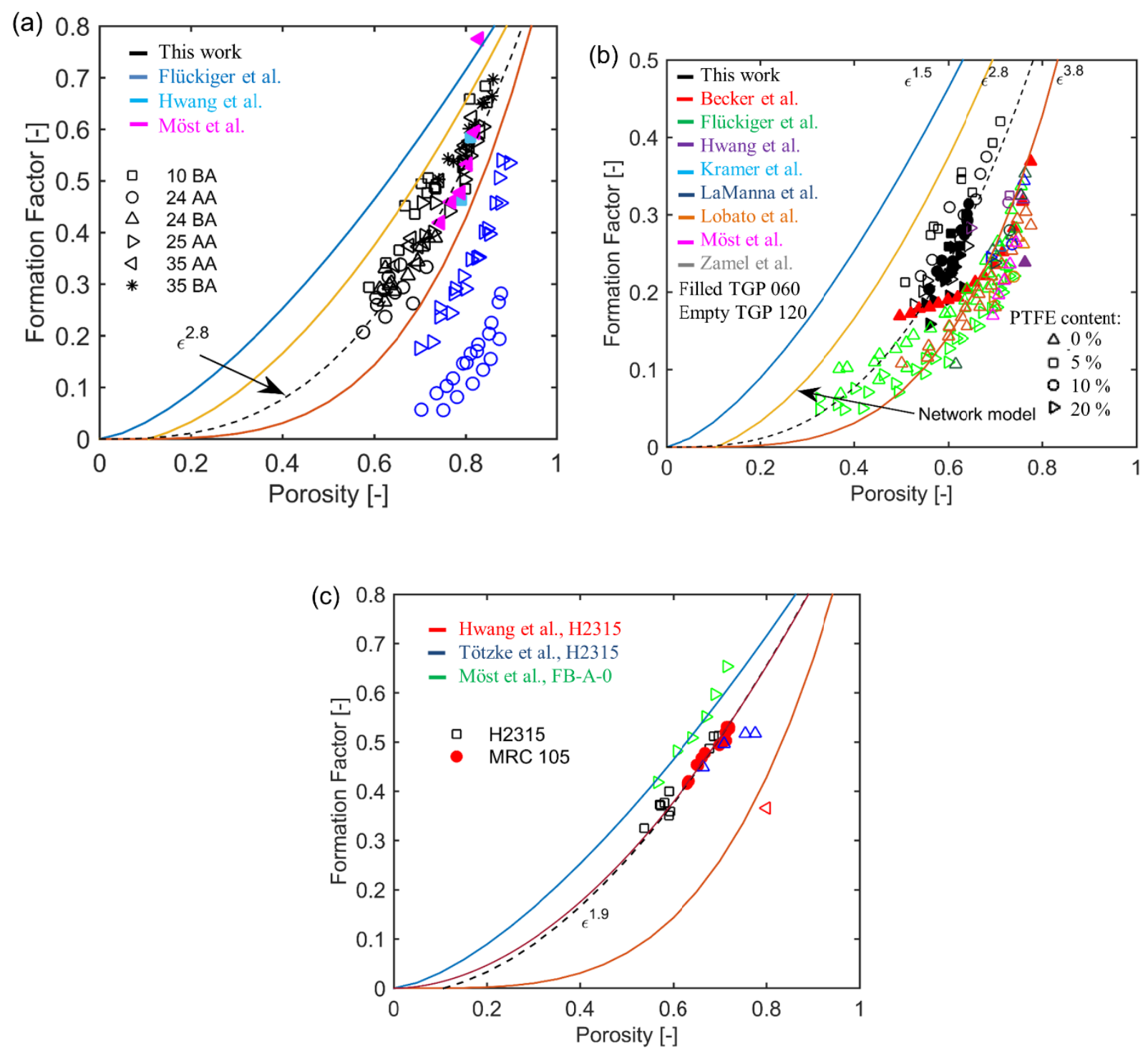

Figure 5Formation factors for a) SGL, b) TGP and c) H2315 and MRC 105 GDLs. The data from this paper is compared to reported literature values from [17-19, 23, 25, 30, 32, 44, 54] .

\subsubsection{Pore Shape}

A decrease in formation factor and increase in tortuosity with compression in the through-plane direction is partially due to preferential fiber orientation in the in-plane direction, binder distribution, and GDL heterogeneity. As previously reported, the through-plane 
tortuosities are about a factor of 2 to 3 larger than those in-plane for TGP and SGL series [23]. However, for Freudenberg GDLs, no significant differences were observed with compression for in-plane versus through-plane effective diffusion coefficients due to the fiber shape and interconnections resulting in a more homogeneous morphology. To explore how GDL's pore shape changes with compression, an ellipsoid-factor $(E F)$ method was used to determine whether pores are rod or plate like [66]. For an object with three semi-axis radii $a, b$ and $c, E F$ is defined as:

$$
a \leq b \leq c, E F=\frac{a}{b}-\frac{b}{c}[10]
$$

From this definition it is evident that $E F$ varies between -1 and 1 , where $E F$ of -1 means that $a / b$ approaches 0 and $b / c$ approaches 1 , which is possible when the object is extremely oblate (discus-shaped) ellipsoid, as shown byFigure 6 . For $E F$ to be $1, b / c$ approaches 0 and $a / b$ approaches 1, where the object is extremely prolate ellipsoid, with one long and two short axes. $E F$ of 0 indicates that the fitted ellipse is a sphere.

Here, EF is used to characterize PSD shapes for SGL 24 BA with 0 and $40 \%$ compression and MRC105 with 0 and 55\% compression as shown by Figure 6a and b, respectively. This figure shows the normalized EF number distribution. For uncompressed SGL 24 BA, a combination of prolate and oblate ellipsoids describes the GDL with an overall wide distribution. Upon compression, the distribution tail shifts towards the left, indicating that now the porous medium has more oblate pores. From a through-plane, effective-transport parameter perspective, having oblate pores is like having GDL represented by slabs, as discussed in [23]. If the end of the pore is blocked by binder or fibers, then the fluid has to travel in the oblate ' $b$ ' 
direction to reach the end of the adjacent pore, and this distance is extremely long, therefore resulting in large tortuosity values and small formation factors.

MRC 105 shows a very unimodal distribution centered around 0.1 to 0.2 ; this distribution is more narrow compared to SGL 24 BA with a clear peak. Uncompressed MRC 105 features primarily circular pores, with a slight inclination towards prolate ellipsoids, indicating 3-D homogeneity. This explains why the effective diffusivity is well approximated by a Bruggeman relation, which assumes domains with spherical pores. Upon 55\% of compression, MRC $105 \mathrm{EF}$ distribution becomes narrower, with a larger peak around 0.1, indicating that the shape of the pores upon compression does not change significantly. Secondly, the pores become closer to spherical as the distribution tail is smaller now and less pores are present with oblate or prolate shapes. These GDLs are expected to have similar effective transport parameters in-plane and through-plane.

Examples of volume-rendered tomographic reconstructions for these two GDLsare shown in Figure $6 \mathrm{c}$ under three levels of compression. Carbon fibers are clearly distinguished from the pore volume, where, for the least compressed (SGL $10 \mathrm{BA}$ ), single fibers protruding outside of the GDL can be seen. SGL 10 BA was subjected to 6,39 and $59 \%$ of compression, where at the highest compression significant reduction in pore-space is observed. The MRC 105 GDL was compressed by 5, 33 and $53 \%$ and volume-rendering also shows a decrease in porosity. 


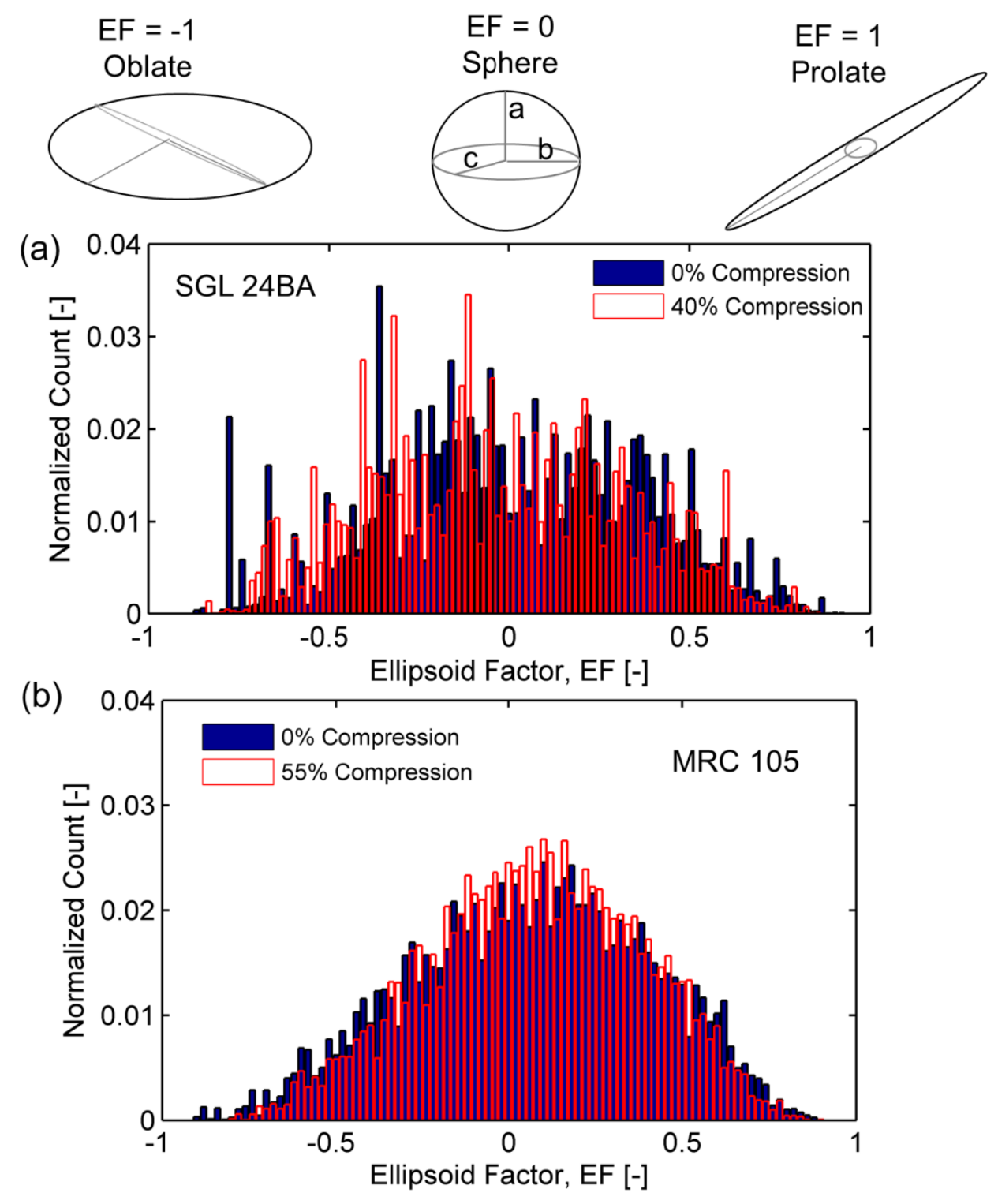



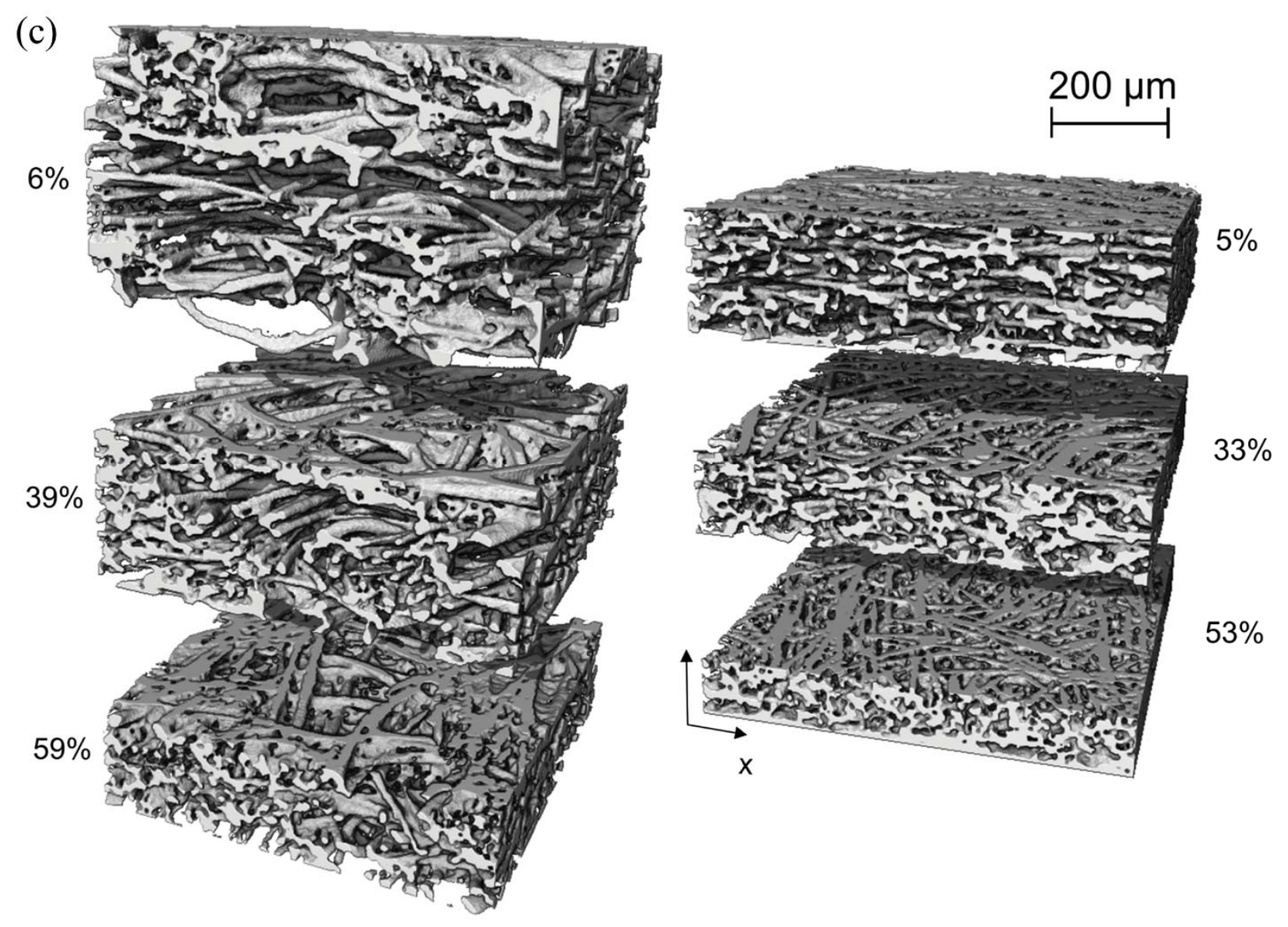

Figure 6. Normalized count as a function of ellipsoid factor, EF for a) SGL 24BA and b) MRC 105 at two compressions. A schematic of representative oblate $(E F=-1)$, spherical $(E F=0)$ and prolate $(E F=1)$ representative inscribed ellipsoids is shown on the top. c) Volume-rendered SGL 10 BA (left) and MRC 105 (right) at three compression levels.

\subsection{Representative elementary volume and heterogeneity}

In image analysis the REV is a critical parameter. It defines not only the statistically representative sample to determine properties, but also the minimum size required that results in the same response; it is a measure of the heterogeneity of the medium. This latter effect is perhaps critical in designing flow-field architectures to ensure predictable and desired response. To evaluate the representative volume, a sample GDL was parsed into 1, 4, 9, or 36 segments, 
each having the same thickness $(350 \mu \mathrm{m})$ but varied length and width of 3, $1.5(\mathrm{~B}), 1$ (C) and 0.5 (D) mm, respectively, as seen in Figure 7a. Then, the PSDs for all the segments were plotted and mean radii for bimodal PSD were compared as shown by Figure $7 \mathrm{~b}$. The average mean radius for elemental volumes B and C did not change significantly and the spread was lower compared to $\mathrm{D}$, where volume to volume mean radius ranged from 10 to $14 \mu \mathrm{m}$ for the smaller radius and 25 to $33 \mu \mathrm{m}$ for the larger one. Moreover, PSDs for volumes D (shown in SI) clearly demonstrated significant deviations from that of $3 \mathrm{~mm}$ domains, and thus were not representative. From the figures, it is observed that for segments greater than $1 \mathrm{~mm}$ or so domains, the curves collapse and it becomes representative (see Figure 7c and Figure 7d). Thus, the minimum REV is around 1 to $1.5 \mathrm{~mm}$ length on a side, which is interesting in that it is similar to the typically channel length in a PEFC. This dimension is similar and slightly larger than that determined through waterbreakthrough experiments, although there it is a bit more indirect since it is a composite throughthickness measurement [64].
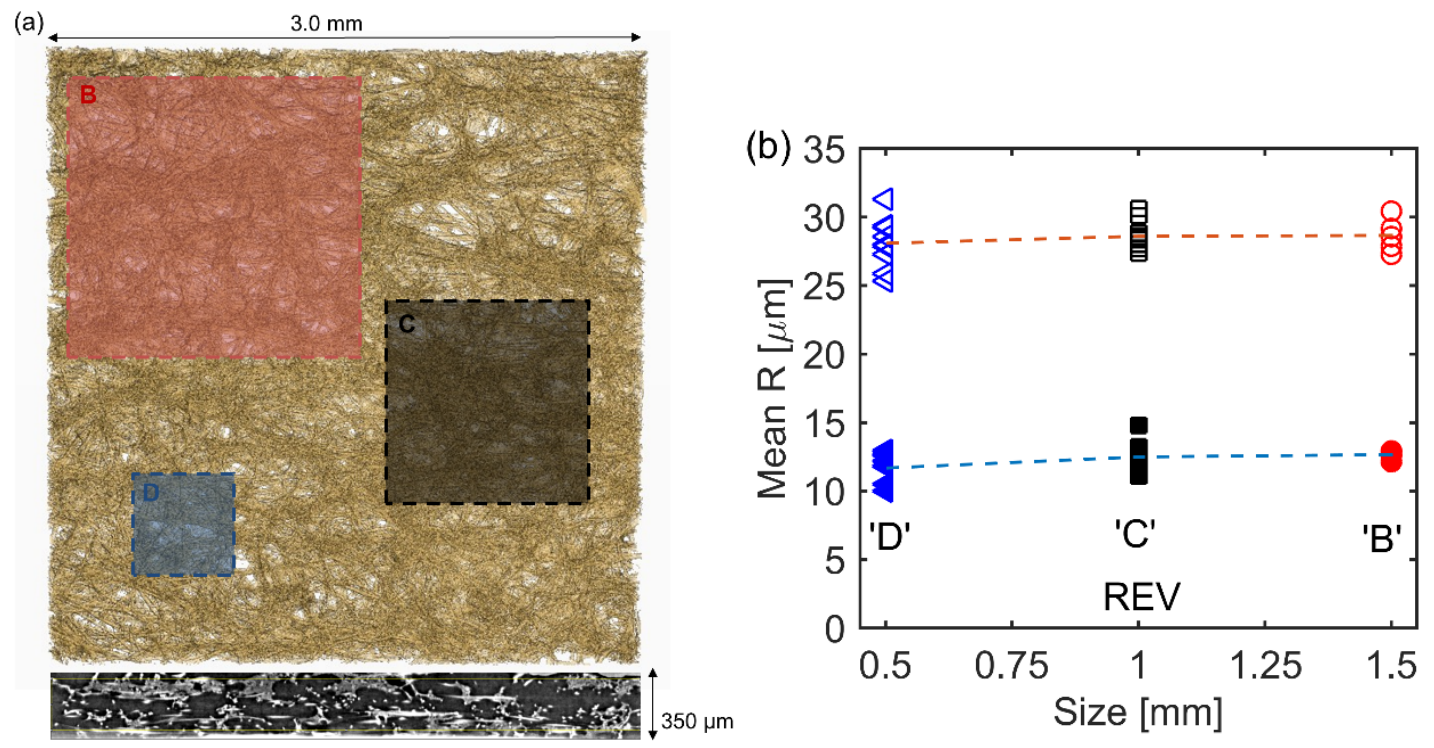

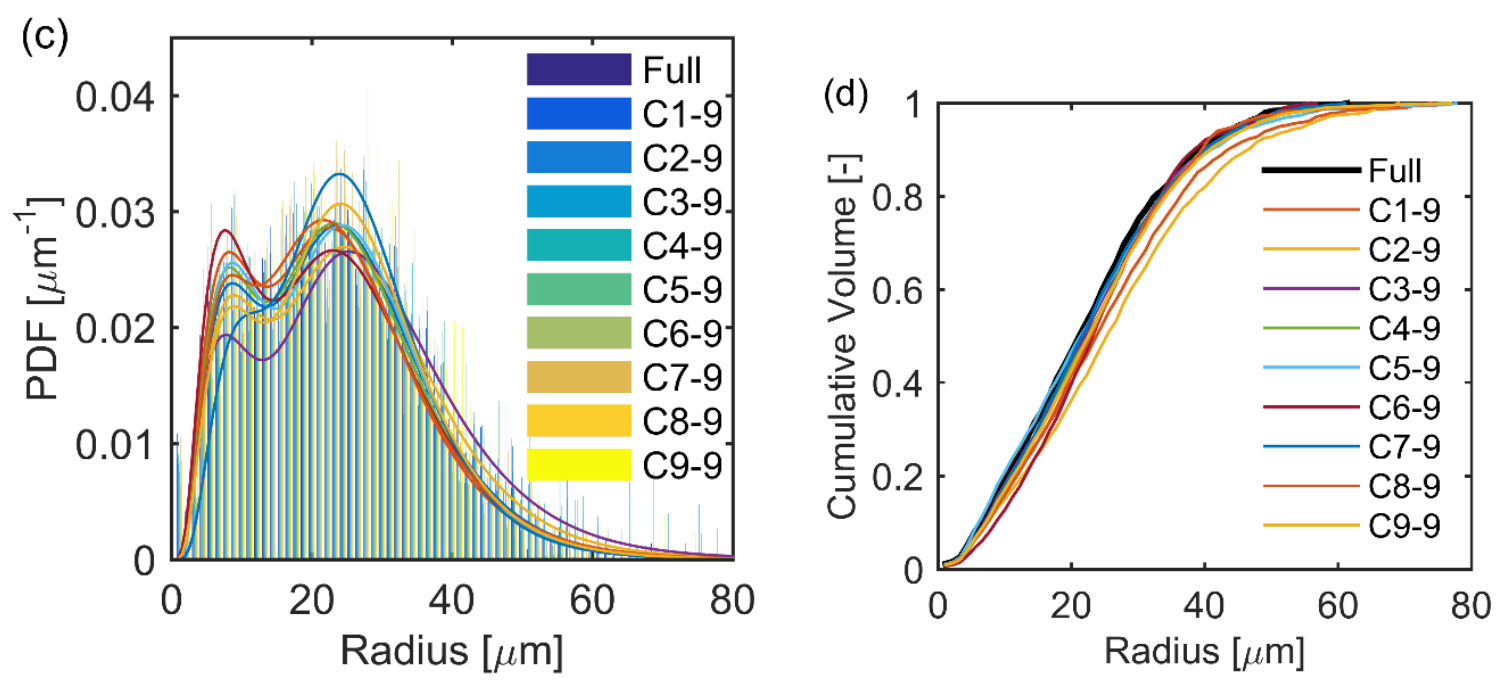

Figure 7.a) Schematic of GDL with three elementary volumes studied of size: $B=1.5 \mathrm{~mm}, \mathrm{C}=1$ $\mathrm{mm}$ and $\mathrm{D}=0.5 \mathrm{~mm}$. b) The two mathematical mean radii of PSD for three elementary volumes, c) The PDF of REV that was found to be $1 \mathrm{~mm}$ for 9 different cuts and c) cumulative volumes.

Similar to the above discussion, one can also do an analysis in the through-thickness direction. However, this is complicated by the natural changes that occur. For example, Figure $8 \mathrm{a}$ shows the porosity as a function of normalized SGL GDL thickness. The data demonstrate a relatively flat profile in Region 2 and a significant valley in Region 1. For MRC 105, shown in Figure $8 \mathrm{~b}$, the porosity profile does not show significant differences in the through-thickness direction, indicating that this material is homogeneous as mentioned above and in agreement with the low tortuosity values calculated.

To understand further the SGL heterogeneous porosity distribution, the PSD for region 1 is shown in Figure 8c. A clear bimodal PSD is observed for this region, which is similar to the overall GDLs' PSD. All eight samples show a very similar large PDF peak at small radius $(\sim 10$ $\mu \mathrm{m})$ and smaller peak for larger mean radius $(\sim 25 \mu \mathrm{m})$. To further understand where the bimodal distribution originates from in terms of GDL structure, a representative Region 1 grey-scale 
tomograph is examined. In Region 1, the fibers and binder are interwoven resulting in a complex porosity and PSD. The large peak on the PDF corresponds to small pores within the binder, whereas the smaller peak corresponds to larger pores between fibers. On the other hand, as shown by Figure 8d, Region 2 does not have significant binder in it, and an almost unimodal distribution with a mean radius of $\sim 20 \mu \mathrm{m}$ is observed. All eight samples show similar PDFs.
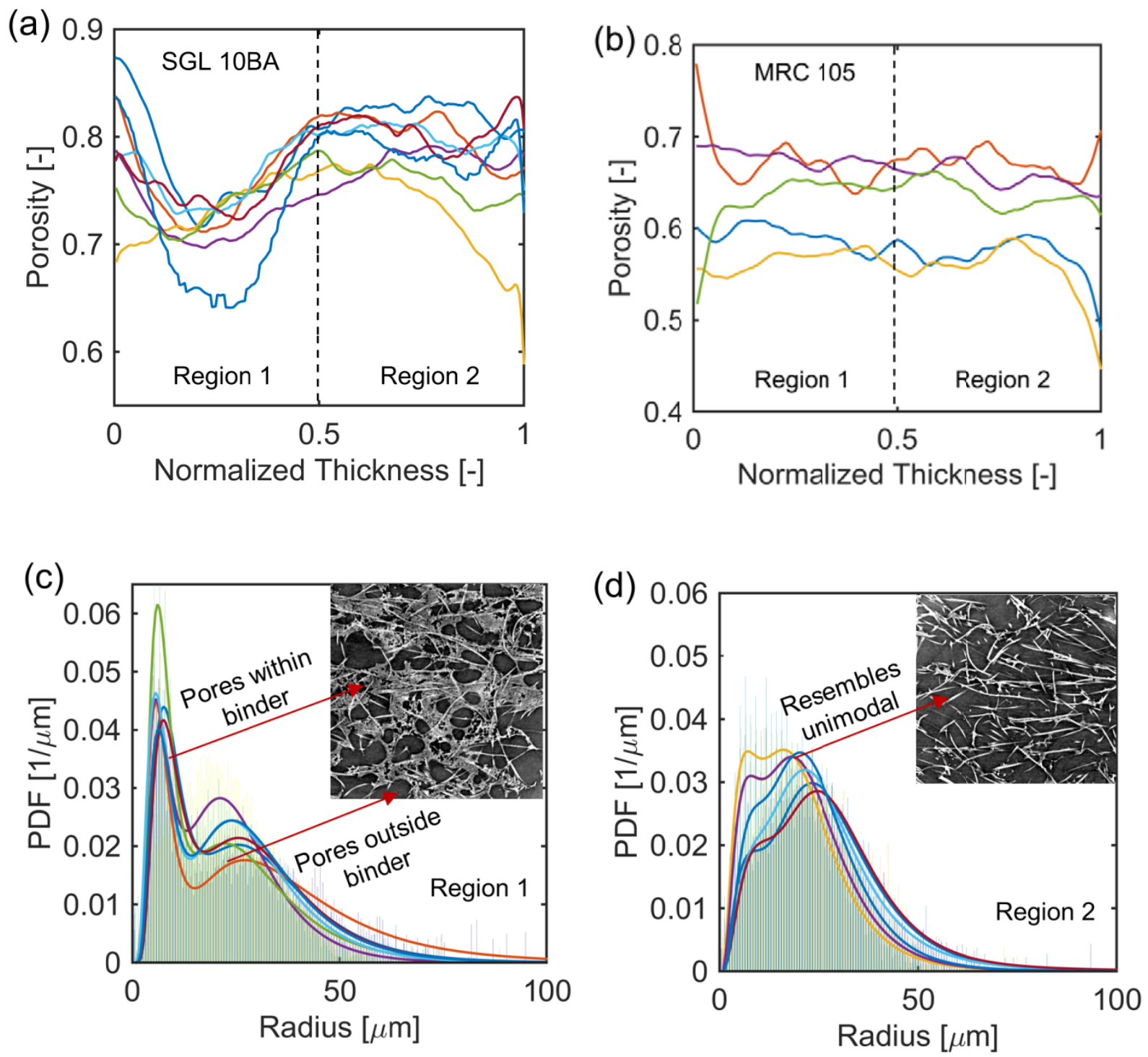

Figure 8. a) Porosity for SGL 10 BA and b) MRC 105 as a function of GDL normalized thickness for eight SGL samples, where Regions 1 and 2 represent first and second half of the 
GDL. PDFs for these eight samples of SGL 10 BA for c) Region 1 and d) Region 2, where insets show sample cross-section tomographs representing these regions.

\section{Summary}

X-ray computed tomography (CT) was used to investigate the morphological properties of commercially available gas-diffusion layers (GDLs) under various levels of compression. Porosity, pore-size distribution (PSD), and tortuosity were reported in this study. X-ray CT is the only viable technique for direct measurement of porosity for compressed GDLs and is a noninvasive measurement. It was found that the porosity decreased for all of the GDLs with compression, and linear fits with various scaling coefficients were determined. In terms of the PSD, for SGL series, the PSD was clearly bimodal with peaks separated by tens of micrometers for uncompressed samples. Upon compression, the peak corresponding to the larger radius shifted to that of the smaller radii, approaching a unimodal distribution. This bimodal distribution was due to smaller pores corresponding to those at the interface between the fibers and binder and where the larger pores are in the regions with minimal binder. TGP series showed also a bimodal distribution but with closer mean radii. Freudenberg H2315 had a unimodal PSD with a mean radius below $10 \mu \mathrm{m}$, whereas MRC had a very similar PSD to H2315 but bimodal. Comparison of the PSDs with those from mercury intrusion porosimetry (MIP) demonstrated that the large tail from MIP was due to interfacial voids.

TGPs and SGLs are the most tortuous and heterogeneous materials compared to MRC and H2315. Overall, excellent agreement with previously reported studies for formation factor and tortuosity are reported here for SGL samples, and deviations from other samples can be explained due to possible inaccurate porosity calculations from those techniques. When 
analyzing the ellipsoid factors for SGL and MRC materials, it was observed that SGL materials have a very heterogeneous structure with a distribution of oblate and prolate ellipsoids, whereas MRC GDL has very rounded pores that maintain their shape even upon compression, indicating very little difference between in-plane and through-plane effective properties. This was further confirmed in the much more heterogeneous through-thickness morphology of SGL compared to MRC, mainly due to binder distribution.

Finally, insight is gained in terms of understanding the critical dimensionality of GDL reproducibility, which is around $1 \mathrm{x} 1 \mathrm{~mm}$ in area, a key metric for both modeling and design. Overall, the analysis conducted provides important expressions (e.g., porosity and PSD as a function of compression) that can be used for modeling and analyzing GDL morphology and its subsequent impact on fuel-cell performance. XCT provides a critical way to analyze the morphology and tortuosity in situations unobtainable by other methods. Future work involves extensions to determining morphologies of partially saturated GDLs under a variety of conditions.

\section{Acknowledgements}

This work was funded under the Fuel Cell Performance and Durability Consortium (FC PAD) funded by the Energy Efficiency and Renewable Energy, Fuel Cell Technologies Office, of the U. S. Department of Energy under contract number DE-AC02-05CH11231, Program Development Manager Dimitrios Papageorgopoulos; and CRADA agreement LB08003874 between LBNL and ToyotaMotor Company. The Advanced Light Source is supported by the 
Director, Office of Science, Office of Basic Energy Sciences, of the U.S. Department of Energy under Contract No. DE-AC02-05CH11231. 


\section{References}

[1] M.K. Debe, Nature, 486 (2012) 43-51.

[2] A.Z. Weber, R.L. Borup, R.M. Darling, P.K. Das, T.J. Dursch, W. Gu, D. Harvey, A.

Kusoglu, S. Litster, M.M. Mench, R. Mukundan, J.P. Owejan, J.G. Pharoah, M. Secanell, I.V.

Zenyuk, J Electrochem Soc, 161 (2014) F1254-F1299.

[3] M.L. Perry, A.Z. Weber, Journal of The Electrochemical Society, 163 (2016) A5064-A5067.

[4] T. Van Nguyen, A. Ahosseini, X. Wang, V. Yarlagadda, A. Kwong, A.Z. Weber, P.

Deevanhxay, S. Tsushima, S. Hirai, Journal of The Electrochemical Society, 162 (2015) F1451F1460.

[5] X. Wei, L. Cosimbescu, W. Xu, J.Z. Hu, M. Vijayakumar, J. Feng, M.Y. Hu, X. Deng, J.

Xiao, J. Liu, V. Sprenkle, W. Wang, Advanced Energy Materials, 5 (2015) n/a-n/a.

[6] A.Z. Weber, M.M. Mench, J.P. Meyers, P.N. Ross, J.T. Gostick, Q. Liu, Journal of Applied Electrochemistry, 41 (2011) 1137-1164.

[7] G.L. Soloveichik, Chemical Reviews, 115 (2015) 11533-11558.

[8] K. Lin, Q. Chen, M.R. Gerhardt, L. Tong, S.B. Kim, L. Eisenach, A.W. Valle, D. Hardee, R.G. Gordon, M.J. Aziz, M.P. Marshak, Science, 349 (2015) 1529-1532.

[9] M.K. Debe, A.J. Steinbach, K. Noda, ECS Transactions, 3 (2006) 835-853.

[10] A.J. Steinbach, M.K. Debe, M.J. Pejsa, D.M. Peppin, A.T. Haug, M.J. Kurkowski, S.M. Maier-Hendricks, ECS Transactions, 41 (2011) 449-457.

[11] A.J. Steinbach, M.K. Debe, J. Wong, M.J. Kurkowski, A.T. Haug, D.M. Peppin, S.K.

Deppe, S.M. Hendricks, E.M. Fischer, ECS Transactions, 33 (2010) 1179-1188.

[12] I.V. Zenyuk, A.Z. Weber, ECS Transactions, 69 (2015) 1253-1265.

[13] I.V. Zenyuk, S. Litster, Electrochimica Acta, 146 (2014) 194-206. 
[14] I.V. Zenyuk, D.Y. Parkinson, G. Hwang, A.Z. Weber, Electrochemistry Communications, 53 (2015) 24-28.

[15] I.V. Zenyuk, R. Taspinar, A.R. Kalidindi, E.C. Kumbur, S. Litster, J Electrochem Soc, 161 (2014) F3091-F3103.

[16] M. Mathias, J. Roth, J. Fleming, W. Lehnert, Handbook of fuel cells, (2003).

[17] R. Flückiger, S.A. Freunberger, D. Kramer, A. Wokaun, G.G. Scherer, F.N. Büchi, Electrochimica Acta, 54 (2008) 551-559.

[18] G.S. Hwang, A.Z. Weber, Journal of the Electrochemical Society, 159 (2012) F683-F692.

[19] J.M. LaManna, S.G. Kandlikar, International Journal of Hydrogen Energy, 36 (2011) 50215029.

[20] R.R. Rashapov, J. Unno, J.T. Gostick, J Electrochem Soc, 162 (2015) F603-F612.

[21] M.M. Tomadakis, S.V. Sotirchos, Chemical Engineering Science, 48 (1993) 3323-3333.

[22] S. Escribano, J.-F. Blachot, J. Ethève, A. Morin, R. Mosdale, Journal of Power Sources, 156 (2006) 8-13.

[23] M. Möst, M. Rzepka, U. Stimming, Journal of Power Sources, 191 (2009) 456-464.

[24] J.T. Gostick, M.A. Ioannidis, M.W. Fowler, M.D. Pritzker, Journal of Power Sources, 194 (2009) 433-444.

[25] J. Lobato, P. Cañizares, M.A. Rodrigo, C. Ruiz-López, J.J. Linares, Journal of Applied Electrochemistry, 38 (2008) 793-802.

[26] A. El-kharouf, T.J. Mason, D.J.L. Brett, B.G. Pollet, Journal of Power Sources, 218 (2012) $393-404$.

[27] M.V. Williams, E. Begg, L. Bonville, H.R. Kunz, J.M. Fenton, J Electrochem Soc, 151 (2004) A1173-A1180. 
[28] J.T. Gostick, M.A. Ioannidis, M.W. Fowler, M.D. Pritzker, Journal of Power Sources, 173 (2007) 277-290.

[29] C. Chan, N. Zamel, X. Li, J. Shen, Electrochimica Acta, 65 (2012) 13-21.

[30] N. Zamel, N.G.C. Astrath, X. Li, J. Shen, J. Zhou, F.B.G. Astrath, H. Wang, Z.-S. Liu, Chemical Engineering Science, 65 (2010) 931-937.

[31] D.R. Baker, D.A. Caulk, K.C. Neyerlin, M.W. Murphy, J Electrochem Soc, 156 (2009) B991-B1003.

[32] D. Kramer, S.A. Freunberger, R. Flückiger, I.A. Schneider, A. Wokaun, F.N. Büchi, G.G. Scherer, Journal of Electroanalytical Chemistry, 612 (2008) 63-77.

[33] M.J. Martínez, S. Shimpalee, J.W. Van Zee, J Electrochem Soc, 156 (2009) B80-B85.

[34] D.R. Baker, C. Wieser, K.C. Neyerlin, M.W. Murphy, ECS Transactions, 3 (2006) 989-999.

[35] J. Becker, R. Flückiger, M. Reum, F.N. Büchi, F. Marone, M. Stampanoni, Journal of The Electrochemical Society, 156 (2009) B1175-B1181.

[36] S. Didari, A. Asadi, Y. Wang, T.A.L. Harris, International Journal of Hydrogen Energy, 39 (2014) 9375-9386.

[37] P.A. Garcia-Salaberri, Hwang G., Vera, M., Weber A.Z., Gostick J.T., Journal of Heat and Mass Transfer, Submitted (2014).

[38] P.A. Garcia-Salaberri, Gostick J.T., Hwang G., Weber A.Z., Vera, M., Journal of Heat and Mass Transfer, Submitted (2015).

[39] T. Rosén, J. Eller, J. Kang, N.I. Prasianakis, J. Mantzaras, F.N. Büchi, J Electrochem Soc, 159 (2012) F536-F544.

[40] T.D. Myles, A.A. Peracchio, U. Pasaogullari, W.K.S. Chiu, J Electrochem Soc, 162 (2015) F645-F650. 
[41] Z. Tayarani-Yoosefabadi, D. Harvey, J. Bellerive, E. Kjeang, Journal of Power Sources, 303 (2016) 208-221.

[42] T.D. Myles, A.A. Peracchio, W.K.S. Chiu, Journal of Applied Physics, 117 (2015) 025101.

[43] P.K. Sinha, P.P. Mukherjee, C.-Y. Wang, Journal of Materials Chemistry, 17 (2007) 30893103.

[44] J. Becker, V. Schulz, A. Wiegmann, Journal of Fuel Cell Science and Technology, 5 (2008) 021006-021006.

[45] I. Manke, C. Hartnig, M. Grünerbel, W. Lehnert, N. Kardjilov, A. Haibel, A. Hilger, J.

Banhart, H. Riesemeier, Applied Physics Letters, 90 (2007) 174105.

[46] F.N. Buechi, R. Flückiger, D. Tehlar, F. Marone, M. Stampanoni, ECS Transactions, 16 (2008) 587-592.

[47] J.r. Becker, R. Flückiger, M. Reum, F.N. Büchi, F. Marone, M. Stampanoni, Journal of The Electrochemical Society, 156 (2009) B1175.

[48] R. Flückiger, F. Marone, M. Stampanoni, A. Wokaun, F.N. Büchi, Electrochimica Acta, 56 (2011) 2254-2262.

[49] J. Eller, T. Rosén, F. Marone, M. Stampanoni, A. Wokaun, F.N. Büchi, Journal of The Electrochemical Society, 158 (2011) B963.

[50] Z. Fishman, J. Hinebaugh, A. Bazylak, Journal of The Electrochemical Society, 157 (2010) B1643.

[51] J. Lee, J. Hinebaugh, A. Bazylak, Journal of Power Sources, 227 (2013) 123-130.

[52] A. Lamibrac, J. Roth, M. Toulec, F. Marone, M. Stampanoni, F.N. Büchi, Journal of The Electrochemical Society, 163 (2016) F202-F209. 
[53] N. Khajeh-Hosseini-Dalasm, T. Sasabe, T. Tokumasu, U. Pasaogullari, Journal of Power Sources, 266 (2014) 213-221.

[54] C. Tötzke, G. Gaiselmann, M. Osenberg, J. Bohner, T. Arlt, H. Markötter, A. Hilger, F.

Wieder, A. Kupsch, B.R. Müller, M.P. Hentschel, J. Banhart, V. Schmidt, W. Lehnert, I. Manke, Journal of Power Sources, 253 (2014) 123-131.

[55] E.F. Medici, Zenyuk, I.V., Parkinson, D.Y., Weber, A.Z., Allen, J.S., Fuel Cells, In Press (2016).

[56] S. Kelly, H. El-Sobky, C. Torres-Verdín, M.T. Balhoff, Advances in Water Resources, (2015).

[57] A. Groso, R. Abela, M. Stampanoni, Opt. Express, 14 (2006) 8103-8110.

[58] I.V. Zenyuk, E.C. Kumbur, S. Litster, Journal of Power Sources, 241 (2013) 379-387.

[59] N. Epstein, Chemical Engineering Science, 44 (1989) 777-779.

[60] T. Bultreys, W. De Boever, V. Cnudde, Earth-Science Reviews, 155 (2016) 93-128.

[61] J.E. McClure, J.F. Prins, C.T. Miller, Computer Physics Communications, 185 (2014) 18651874.

[62] C. Manwart, U. Aaltosalmi, A. Koponen, R. Hilfer, J. Timonen, Physical Review E, 66 (2002) 016702.

[63] X. Yang, Y. Mehmani, W.A. Perkins, A. Pasquali, M. Schönherr, K. Kim, M. Perego, M.L. Parks, N. Trask, M.T. Balhoff, M.C. Richmond, M. Geier, M. Krafczyk, L.-S. Luo, A.M. Tartakovsky, T.D. Scheibe, Advances in Water Resources.

[64] A.D. Santamaria, P.K. Das, J.C. MacDonald, A.Z. Weber, J Electrochem Soc, 161 (2014) F1184-F1193.

[65] Z. Fishman, A. Bazylak, J Electrochem Soc, 158 (2011) B247-B252. 
[66] M. Doube, Frontiers in Endocrinology, 6 (2015).

[67] Y. Lee, B. Kim, Y. Kim, X. Li, Applied Energy, 88 (2011) 5111-5119.

[68] D. Cheng, S. Ye, E. Gyenge, Electrochemical and Solid-State Letters, 11 (2008) B148-

B152.

[69] J.P. Owejan, T.A. Trabold, M.M. Mench, International Journal of Heat and Mass Transfer, $71(2014) 585-592$.

[70] J.T. Gostick, M.W. Fowler, M.D. Pritzker, M.A. Ioannidis, L.M. Behra, Journal of Power Sources, 162 (2006) 228-238.

[71] H. Kim, Y.-J. Lee, S.-J. Lee, Y.-S. Chung, Y. Yoo, J Mater Sci, 49 (2014) 3831-3838. 
Table 1. GDLs measured thicknesses and porosity compared to the previously reported literature values.

\begin{tabular}{|c|c|c|c|c|c|c|}
\hline Name & $\begin{array}{l}\text { Thickness } \\
{[\mu \mathrm{m}]}\end{array}$ & $\begin{array}{l}\text { St. } \\
\text { Deviation } \\
{[\mu \mathrm{m}]}\end{array}$ & $\begin{array}{l}\text { Reported } \\
{[\mu \mathrm{m}]}\end{array}$ & Compression & Porosity & Reported \\
\hline $\begin{array}{l}\text { TGP120- } \\
\text { 20PTFE }\end{array}$ & 360 & 4.7 & $365[20]$ & 1 & 0.62 & $\begin{array}{l}0.719[20], 0.696[25], \\
0.649[64], 0.64[53]\end{array}$ \\
\hline $\begin{array}{l}\text { TGP120- } \\
\text { 10PTFE }\end{array}$ & 370 & 5.1 & $362[20]$ & 8.4 & 0.63 & $\begin{array}{l}0.74[25], 0.728[64], \\
0.662[53], 0.73[20]\end{array}$ \\
\hline $\begin{array}{l}\text { TGP120- } \\
\text { 5PTFE }\end{array}$ & 386.7 & 4.71 & 364 & 15.5 & 0.626 & 0.73 [64], 0.746 [20] \\
\hline $\begin{array}{l}\text { TGP060- } \\
\text { 20PTFE }\end{array}$ & 205 & 5.1 & 186 & 8.8 & 0.627 & $\begin{array}{l}0.75[48], 0.71[67], \\
0.74[48], \\
0.67[20]\end{array}$ \\
\hline $\begin{array}{l}\text { TGP060- } \\
\text { 10PTFE }\end{array}$ & 205.5 & 7.6 & 188 & 13.1 & 0.64 & $0.738[20]$ \\
\hline $\begin{array}{l}\text { TGP060- } \\
\text { 5PTFE }\end{array}$ & 226 & 6 & $178[20]$ & 7.1 & 0.69 & $0.734[20], 0.62[68]$ \\
\hline $\begin{array}{l}\text { TGP060- } \\
\text { 5PTFEMPL }\end{array}$ & 196.8 & 11 & $225[69]$ & 0 & & \\
\hline SGL10BA & 440 & 21 & $\begin{array}{l}432[20], \\
370[29]\end{array}$ & 6.1 & 0.82 & $\begin{array}{l}0.87[20], 0.88[70], \\
0.84[67]\end{array}$ \\
\hline SGL10BC & 382.5 & 10.9 & $420[19]$ & 15 & & \\
\hline SGL24AA & 205 & 5 & $187[20]$ & 4.7 & 0.7 & $\begin{array}{l}0.74[48], 0.78[71], \\
0.85[20]\end{array}$ \\
\hline SGL24BA & 210 & 5.3 & $198[20]$ & 1.7 & 0.77 & $\begin{array}{l}0.81[70], 0.74[26], \\
0.85[20]\end{array}$ \\
\hline SGL24BC & 242.5 & 4.3 & & 8.3 & & \\
\hline SGL25AA & 192.2 & 4.1 & 191 & 4.6 & 0.81 & $0.88[20]$ \\
\hline SGL25BC & 209.6 & 5.2 & $225[19]$ & 11 & & \\
\hline
\end{tabular}




\begin{tabular}{|c|c|c|c|c|c|c|}
\hline \multirow[t]{2}{*}{ SGL35AA } & 332.6 & 9 & $255[20]$ & 2.1 & 0.82 & $0.88[20], 0.84[23]$ \\
\hline & & & $300[54]$ & & & \\
\hline SGL35BA & 325 & 11 & $280[20]$ & 0 & 0.85 & $\begin{array}{l}0.89[20], 0.70 \\
{[26]}\end{array}$ \\
\hline SGL35BC & 346.2 & 12.85 & $325[19]$ & 3.8 & & \\
\hline $\mathrm{H} 2315$ & 223 & 7 & 223 [54] & 11.2 & 0.685 & $\begin{array}{l}0.78 \\
{[54],} \\
0.687 \\
{[20]}\end{array}$ \\
\hline MRC105 & 215 & 5.1 & $210[19]$ & 5.1 & 0.72 & \\
\hline
\end{tabular}


Table 2. GDLs' extrapolated porosity at zero compression and parameters for Eqs. [4], [6] and [7].

\begin{tabular}{lrrrrrr}
\hline Name & $\begin{array}{l}\text { Extrapolated } \\
\text { porosity, } \varepsilon_{0}\end{array}$ & Slope, $a$ & $\begin{array}{c}r_{0, \text { mean }}, \\
{[\mu \mathrm{m}]}\end{array}$ & $\begin{array}{l}a_{\text {mean }}, \\
{\left[\mu \mathrm{m}^{-1}\right]}\end{array}$ & $\begin{array}{l}r_{0, \max }, \\
{[\mu \mathrm{m}]}\end{array}$ & $a_{\max },\left[\mu \mathrm{m}^{-1}\right]$ \\
\hline TGP120-20PTFE & 0.62 & 0.76 & 14.1 & 13.6 & 42.8 & 68.2 \\
TGP120-10PTFE & 0.65 & 0.67 & 13.3 & 11.4 & & \\
TGP120-5PTFE & 0.64 & 0.28 & 13.3 & 6.9 & 30.5 & 12.3 \\
TGP060-20PTFE & 0.69 & 1.0 & 13.7 & 10.5 & 29.4 & 0.40 \\
TGP060-10PTFE & 0.70 & 1.54 & 15.9 & 26.6 & 37.4 & 40.4 \\
TGP060-5PTFE & 0.71 & 1.77 & 17.2 & 23.1 & 46.4 & 63.4 \\
SGL10BA & 0.84 & 1.52 & 35.0 & 40.7 & 115.7 & 98.1 \\
SGL24AA & 0.73 & 1.76 & 26.3 & 38.4 & 89.1 & 168 \\
SGL24BA & 0.76 & 2.18 & 27.7 & 36.1 & 79.3 & 102.9 \\
SGL25AA & 0.80 & 1.39 & 32.2 & 41.8 & 85.9 & 81.8 \\
SGL35AA & 0.84 & 2.99 & 29.9 & 28.0 & 87.7 & 57.8 \\
SGL35BA & 0.84 & 1.94 & 33.8 & 32.9 & 93.8 & 82.5 \\
H2315 & 0.71 & 1.74 & 12.4 & 8.81 & 38.6 & 28.1 \\
MRC105 & 0.73 & 0.99 & 11.9 & 18.8 & 53.7 & 115.3 \\
\hline
\end{tabular}


Table 3. Parameters for bimodal log-normal PSDs.

\begin{tabular}{|c|c|c|c|c|c|c|c|}
\hline Name & Strain & $\begin{array}{c}\sigma_{1} \\
{[\mu \mathrm{m}]}\end{array}$ & $\begin{array}{c}\sigma_{2} \\
{[\mu \mathrm{m}]}\end{array}$ & $\begin{array}{c}r_{o, 1} \\
{[\mu \mathrm{m}]}\end{array}$ & $r_{o, 2}[\mu \mathrm{m}]$ & $f_{r, 1}$ & $\mathrm{R}^{2}$ \\
\hline \multirow[t]{4}{*}{ SGL 25 AA } & 0 & 0.768 & 0.401 & 13.73 & 39.8 & 0.335 & 0.919 \\
\hline & 0.046 & 0.758 & 0.388 & 13.53 & 37.0 & 0.379 & 0.941 \\
\hline & 0.175 & 0.754 & 0.344 & 14.09 & 29.5 & 0.498 & 0.933 \\
\hline & 0.335 & 0.680 & 0.304 & 12.19 & 25.2 & 0.598 & 0.969 \\
\hline \multirow{5}{*}{$\begin{array}{l}\text { TGP120 } 20 \% \\
\text { PTFE }\end{array}$} & 0.008 & 0.491 & 0.261 & 10.06 & 15.1 & 0.428 & 0.924 \\
\hline & & & & & & & \\
\hline & 0.056 & 0.449 & 0.247 & 9.57 & 14.7 & 0.426 & 0.928 \\
\hline & 0.061 & 0.466 & 0.245 & 9.85 & 14.7 & 0.460 & 0.962 \\
\hline & 0.237 & 0.436 & 0.245 & 9.27 & 13.7 & 0.500 & 0.946 \\
\hline \multirow[t]{4}{*}{ Н 2315} & 0.112 & 0.541 & - & 9.50 & - & 1 & 0.940 \\
\hline & 0.130 & 0.490 & - & 8.04 & - & 1 & 0.983 \\
\hline & 0.175 & 0.470 & - & 7.76 & - & 1 & 0.915 \\
\hline & 0.245 & 0.439 & - & 7.28 & - & 1 & 0.977 \\
\hline \multirow[t]{7}{*}{ MRC 105} & 0.051 & 0.484 & 0.296 & 9.55 & 13.09 & 0.611 & 0.952 \\
\hline & 0.153 & 0.457 & 0.282 & 9.01 & 12.98 & 0.597 & 0.983 \\
\hline & 0.247 & 0.291 & 0.449 & 12.39 & 8.62 & 0.439 & 0.941 \\
\hline & 0.330 & 0.217 & 0.436 & 11.56 & 8.72 & 0.238 & 0.982 \\
\hline & 0.345 & 0.411 & - & 9.29 & - & 1 & 0.964 \\
\hline & 0.428 & 0.334 & 0.447 & 8.86 & 5.84 & 0.746 & 0.977 \\
\hline & 0.540 & 0.383 & - & 7.37 & - & 1 & 0.893 \\
\hline
\end{tabular}

Table 4. Formation factors in this study and extrapolated from literature. 


\begin{tabular}{lrl}
\hline Name & Formation Factor Exponent & Reported \\
\hline TGP120-20PTFE & 3 & \\
TGP120-10PTFE & 2.76 & \\
TGP120-5PTFE & 2.74 & \\
TGP120-0PTFE & & $4.04[19], 4.23[32], 4.5[23]$ \\
TGP060-20PTFE & 2.81 & $4.4[17]$ \\
TGP060-10PTFE & 2.6 & \\
TGP060-5PTFE & 2.36 & \\
TGP060- 0PTFE & & $3.6[35], 3.75[17]$ \\
SGL10BA & 2.28 & \\
SGL24AA & 2.8 & $9.7[17]$ \\
SGL24BA & 2.75 & \\
SGL25AA & 2.74 & $5.2[17]$ \\
SGL35AA & 2.6 & $2.66[23]$ \\
SGL35BA & 2.39 & \\
H2315 & 1.83 & $1.45[23], 2.2[54]$ \\
MRC105 & 1.9 & \\
\hline
\end{tabular}




\section{Figure Captions:}

Figure 1 a) A schematic of sample-holder set-up at the beamline and b) a photograph of the sample holder mounted on the stage.

Figure 2. Porosity as a function of compression (strain) for a) SGL, b) Toray and c) H2315 and MRC 105 GDLs. The dashed lines are fits to the experimental data using Eq. 6 as reported in Table 2.

Figure 3. PSDs for different levels of compression for a) SGL 25AA, b) TGP120 20\% PTFE,c) $\mathrm{H} 2315$, d) MRC 105, where $\triangle \mathrm{PDF}$ plots represent the difference in the volume between the least compressed and that at higher compressions.

Figure 4 Mean radii from PSDs for a) SGL, b) TGP and c) H2315 and MRC 105 GDLs as functions of strain. Solid symbols show GDLs with MPLs. Linear regression fits are also shown, where the slope and intercept are reported by Table 2 .

Figure 5Formation factors for a) SGL, b) TGP and c) H2315 and MRC 105 GDLs. The data from this paper is compared to reported literature values from $[17-19,23,25,30,32,44,54]$.

Figure 6. Normalized count as a function of ellipsoid factor, EF for a) SGL 24BA and b) MRC 105 at two compressions. A schematic of representative oblate $(E F=-1)$, spherical $(E F=0)$ and prolate $(E F=1)$ representative inscribed ellipsoids is shown on the top. c) Volume-rendered SGL 10 BA (left) and MRC 105 (right) at three compression levels.

Figure 7.a) Schematic of GDL with three elementary volumes studied of size: $\mathrm{B}=1.5 \mathrm{~mm}, \mathrm{C}=1$ $\mathrm{mm}$ and $\mathrm{D}=0.5 \mathrm{~mm}$. b) The two mathematical mean radii of PSD for three elementary volumes, c) The PDF of REV that was found to be $1 \mathrm{~mm}$ for 9 different cuts and c) cumulative volumes.

Figure 8. a) Porosity for SGL 10 BA and b) MRC 105 as a function of GDL normalized thickness for eight SGL samples, where Regions 1 and 2 represent first and second half of the GDL. PDFs for these eight samples of SGL 10 BA for c) Region 1 and d) Region 2, where insets show sample cross-section tomographs representing these regions. 